\title{
A study on the relationship between the resorption of upper and lower deciduous canines in patients with impacted permanent canines
}

\author{
Hristina Arnautska
}

Department of Prosthetic Dental Medicine and Orthodontics, Faculty of Dental Medicine, Medical University Varna

\begin{abstract}
Delayed eruption of deciduous teeth and continuous formation of the roots of permanent upper canines frequently lead to an altered eruption path of the canines and their palatal impaction.

The aim of the present paper was to examine the relationship between deciduous canines' resorption and the eruption of permanent ones in patients with impacted canines. The patients under study were divided into two groups. In the clinical group, 196 panoramic radiographs were retrospectively examined, involving 98 patients with complete permanent dentition with presence of impacted permanent canines, with complete root development. In the control group, 182 panoramic radiographs were examined in the period of dentition formation, comprising 70 patients with all permanent teeth erupted, with complete root development. In order to determine the extent of resorption of persistent upper temporary canines, Tagychi's methodology was applied, modified by the researchers of this study.

Monitoring teeth eruption and reporting deviations in the resorption of temporary teeth were seen as preventive steps with great importance for the impaction of permanent canines. Where there was impaction tendency for upper and lower canines from the beginning of late mixed dentition the study reported delayed resorption of upper temporary canines, persistent lower temporary canines as well as eruption deviation in permanent canines, which were all absent in a normally developing dentition.
\end{abstract}

Keywords: impacted canine, resorption, deciduous canine, delayed eruption 


\section{Introduction}

Delayed eruption of deciduous teeth and continuous formation of the roots of permanent upper canines frequently lead to an altered eruption path of the canines and their palatal impaction [1]. These complexities related to the eruption of canines had become the objective of the present study on the relationship between the eruption paths of canines in both dental arches. An essential preventive approach was to monitor the deviations in the dental age of each patient which in turn enabled clinicians to overcome impaction tendencies.

At the start of 8th year of a patient, in normal developing dentition, the upper canine tooth could be palpated highly in the vestibular floor of the nose, above the space where it would emerge in the dental arch. This position was clinically observed at 9th year. Half a year later, fourth teeth erupted and 2/3 of the canine root was already formed, while the resorption of temporary canines had advanced to $1 / 2$ of their root's length. Close to 10th-11th year there was already a lower and better shaped vestibular convexity, and by 11 th-12th year there were early signs of eruption of upper canines $[2,3,4]$

Literature sources referred to the age of 6 as the time when calcification of the lower canine crown was complete and although it was located farthest from the occlusal plane, the lower canine erupted around 9th year, doing so ahead of upper canines by about 2 years $[4,5]$.

According to some authors $[1,2,6]$ the lack of resorption of the temporary canine was a major etiologic factor for the path change of the erupting permanent canine. Other authors $[1,7,8]$ opposed this opinion indicating that the altered eruption path of the permanent canine was indeed the reason for the lack of resorption of the root of the temporary canine. Becker [9] held the view that there might be several types of movement of the canine: some were genetically controlled, while others were not.

Regardless of the reason, the presence of persistent temporary canines and the deviation in eruption time of the permanent canine $[10,11]$ was a clinical indicator for an impacted permanent canine.

\section{Aim}

The aim of the present paper was to examine the relationship between deciduous canines' resorption and the eruption of permanent ones in patients with impacted canines.

\section{Material and Methods}

378 panoramic radiographs (OPGs) of 168 patients were analyzed from the database of the Department of Orthodontics at the Dental Faculty of Sofia, the Dental Faculty of Varna as well as from the Private Dental Practice of the researcher.

The patients under study were divided into two groups:

In the clinical group,196 OPGs were retrospectively examined, involving 98 patients with complete permanent dentition, with no eruption of wisdom teeth and with presence of impacted upper and single lower permanent canines, with complete root development. 
In the control group,182 OPGs were examined in the period of dentition formation, comprising 70 patients with all permanent teeth erupted, with complete root development, without eruption of wisdom teeth. In order to determine the extent of resorption of persistent upper temporary canines, Tagychi's methodology [12] was applied, modified by the researchers of this study, aiming to measure the resorption of the lower and upper temporary canines, using the 5-point scale of resorption phases of temporary canines (Fig. 1):

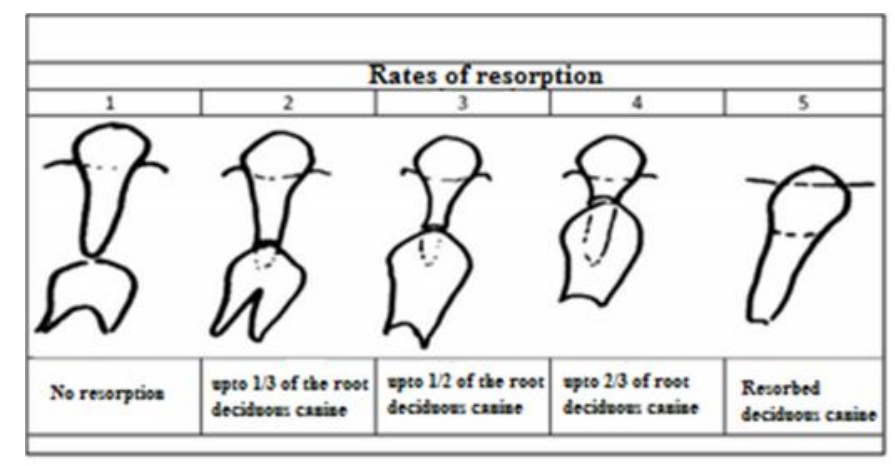

Figure 1. Rates of resorption of the temporary upper canine

\section{Results}

The findings of this study on the relationship between the eruption of lower and upper canines revealed that for the clinical group, with patients in early mixed dentition, $63.6 \%$ of them demonstrated lower temporary canines resorbing and falling out before upper canines. In $31.8 \%$ of the cases symmetrical resorption was observed; in $4.6 \%$ of them the upper temporary canines resorbed earlier than the lower canines, which was indicative of the delayed resorption of the whole dentition in the clinical group.

In the control group the resorption of lower deciduous teeth in $57.5 \%$ of the cases happened earlier than the resorption of upper temporary canines; in $40.6 \%$ it occurred symmetrically and in only $1.9 \%$ of the cases the upper temporary canines resorbed earlier than lower canines (Fig.2). There was no statistically significant difference between the two groups which could be explained by the deep position of the canine germs in both jaws at the time. 


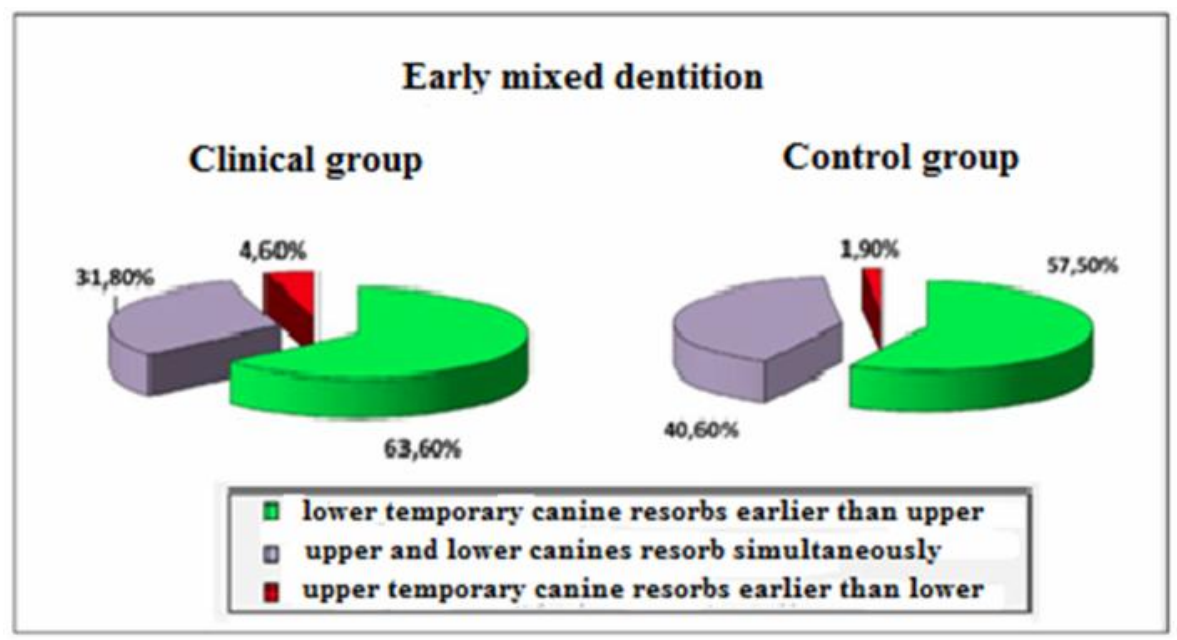

Figure 2. Percentage distribution of the relationship between the resorption of upper temporary canine and lower temporary canine in early mixed dentition

In the clinical group, with patients in phase 1 late mixed dentition (erupting lower canines and upper first premolars) it was established that for $66.60 \%$ of them the lower temporary canine resorbed earlier than its upper counterpart while for $2.80 \%$ of the patients the resorption of the upper temporary canine happened earlier than the resorption of the lower temporary canine, which is associated with the altered eruption path of lower canines. In $30.60 \%$ of the cases upper and lower temporary canines resorbed simultaneously (Fig.3).

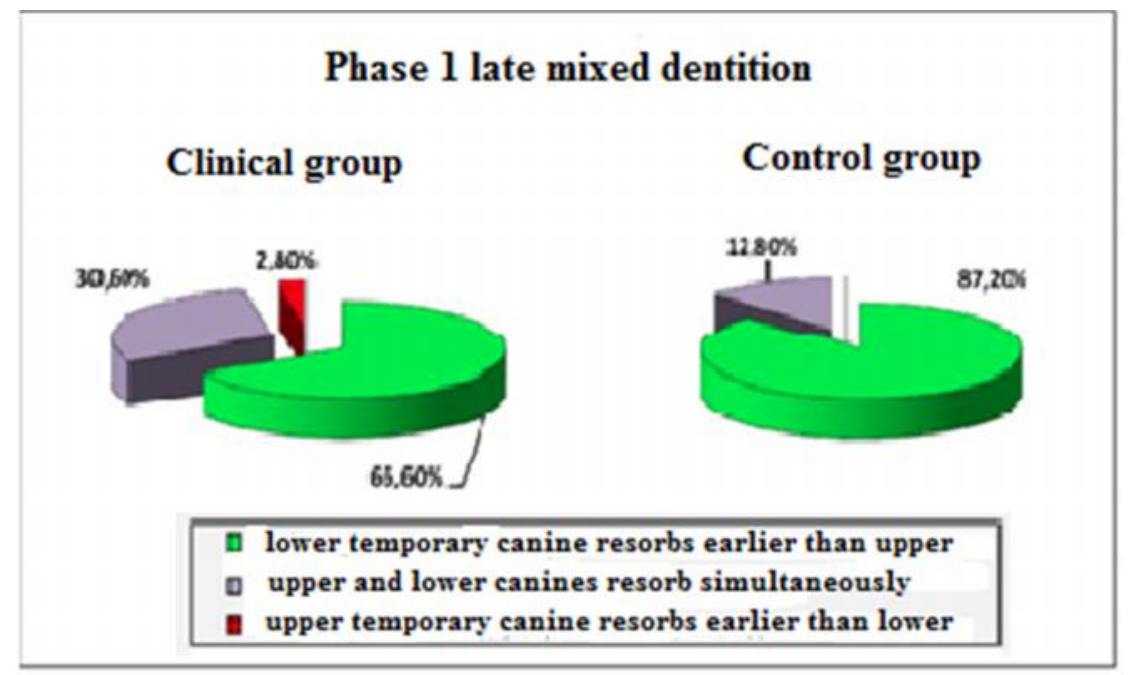

Figure 3. Percentage distribution of the relationship between the resorption of upper temporary canine and lower temporary canine in phase 1 late mixed dentition

In the control group, in $87.20 \%$ of the cases lower canines had already resorbed earlier than upper canines; in only $12.80 \%$ they resorbed simultaneously. The statistically significant difference was evident 
$(P<0.05)$. In the normally developing dentitions the lowest temporary canines had fallen out and there were single teeth with pending resorption.

In the clinical group, with patients in phase 2 late mixed dentition (erupting upper and lower second premolar and upper canines) the observations showed that in $73.80 \%$ of the cases lower deciduous canines had fallen out. For $23.80 \%$ of the patients the resorption of upper and lower temporary canines occurred symmetrically, which was associated with generally delayed dental development of impacted upper canines. In $2.4 \%$ of temporary canines the resorption in the upper jaw occurred earlier than the resorption in the lower jaw, i.e. there were persistent lower temporary canines due to the presence of impacted lower canines (Fig.4).

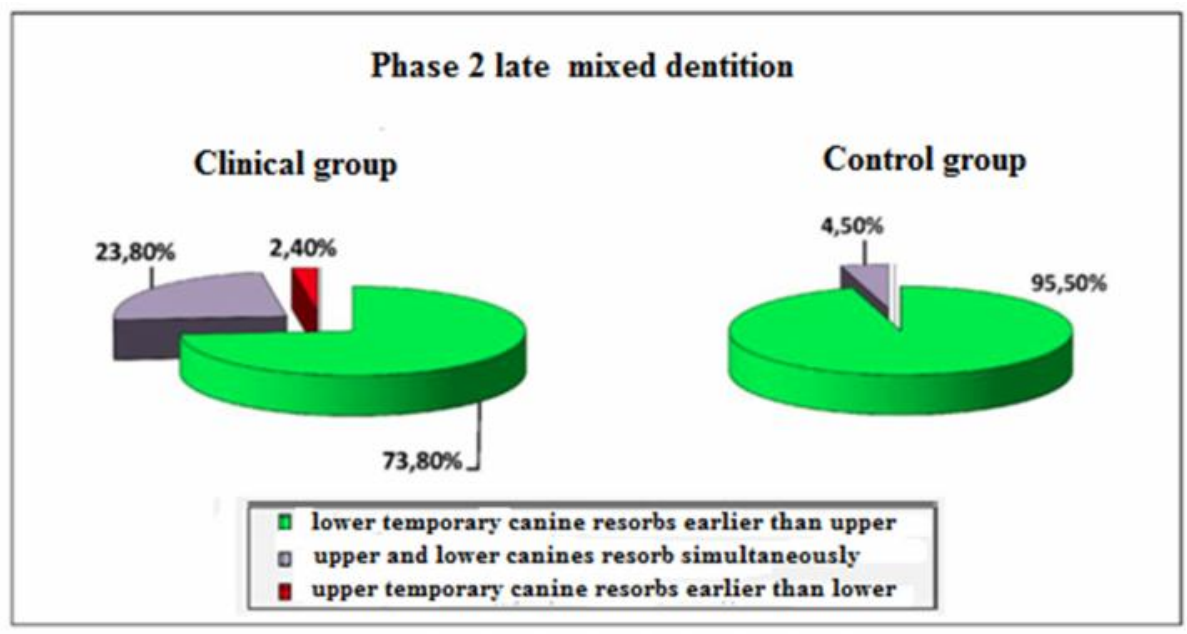

Figure 4. Percentage distribution of the relationship between the resorption of upper temporary canine and lower temporary canine in phase 2 late mixed dentition

In the control group, in $95.5 \%$ of the cases the permanent lower canine tooth had erupted before the upper canines. The statistically significant difference was apparent $(P<0.05)$. In the control group, normally developing dentitions were established with no cases of persistent lower canines.

\section{Discussion}

From a pathogenic viewpoint the earlier eruption of the lower canines was an objective indicator for a normally developing dentition. Any delay in the eruption of lower canines was indicative of a potential deviation in the eruption path and it was most necessary to apply timely preventive measures to avoid impaction of lower canines. Furthermore, the delayed resorption of lower deciduous canines might be linked to impacted upper canines [13,14].

The results of the present study showed that the clinical group displayed delayed resorption of upper and lower temporary canines, whereas the control group lacked any time deviations from a normal developing dentition. 
Earlier resorption of upper canines was observed in $4.6 \%$ of the cases of impacted lower canines, in early mixed dentition. Hence, the impaired resorption in the lower jaw, when upper canines erupted before lower canines, was linked to the impaction of the permanent canines.

In the clinical group with patients in phase 2 late mixed dentition, the impaction of upper canines caused delayed eruption and retention of lower canines. The resorption of temporary canines in the upper and lower jaw occurred symmetrically in $23.8 \%$ of all canines, which signified for an overall slow dental development. In $2.4 \%$ of the cases the presence of a persistent lower temporary canine and the earlier resorption of the upper temporary canine indicated an impaired resorption and an altered eruption path of the lower permanent canine as well.

The control group demonstrated no deviations in the time and sequence of falling out of the temporary canines and in the eruption of permanent canines and normal developing dentition was observed.

\section{Conclusion}

Monitoring teeth eruption and reporting deviations in the resorption of temporary teeth were seen as preventive steps with great importance for the impaction of permanent canines. The patient's age at the time of detection of any deviations in the eruption paths as well as the patient's timely referral for treatment was the most significant factor for the outcome of treatment. Where there was impaction tendency for upper and lower canines from the beginning of late mixed dentition, the study reported delayed resorption of upper temporary canines, persistent lower temporary canines as well as eruption deviation in permanent canines, which were all absent in a normally developing dentition.

\section{References}

1. Newcomb MR. Recognition and interception of aberrant canine eruption. Angle Orthod. 29, 1959.

2. Broadbent BH. Ontogenetic development of occlusion. October, 1941.

3. Korbendau JM, Patti A. Clinical success in surgical and orthodontic treatment of impacted teeth. Paris, Quintessence, 2006.

4. Moyers RE. Handbook of orthodontics. 4th ed. Chicago, Year Book Medical Publishers Inc., 1988, p.140.

5. Van der Linden FPGM, Duterloo HS. Development of the human dentition: an atlas. Hagerstown, MD, Harper\&Row, 1976.

6. Lappin MM. Practical management of the impacted maxillary cuspid. Am. J. Orthod. 37, 1951, No $10,769-778$.

7. Jacobs SG. Reducing the incidence of unerupted palatally displaced canines by extraction of deciduous canines: the history and application of this procedure with some case reports. Aust. Dent. J. 43, 1998, No 1, 20-27. 
8. Schmuth GPF, Freisfeld M, Köster O, Schüller H. The application of computerized tomography (CT) in cases of impacted maxillary canines. Eur. J. Orthod. 14, 1992, No 4, 296-301.

9. Becker A. In defense of the guidance theory of palatal canine displacement. Angle Orthod. 65, 1995, No 2, 95-98.

10. Bishara SE. Impacted maxillary canines: a review. Am. J. Orthod. Dentofacial Orthop.,101, 1992, No 2, 159-171.

11. Thilander B, Jakobsson SO. Local factors in impaction of maxillary canines. Acta Odontol. Scand. 26, 1968, No 2, 145-168.

12. Taguchi $Y$, Hayashi-Sakai S, Tsuda T. Severe cases of ectopically erupting maxillary canine with excessive mesial angulation. Pediatr. Dent. J. 18, 2008, No 1, 34-42.

13. Anic-Milosevic S, Varga S, Mestrovic S, Lapter-Varga M, Slaj M. Dental and occlusal features in patients with palatally displaced maxillary canines. Eur. J. Orthod. 31, 2009, No 4, 367-373.

14. Rozylo-Kalinowska I, Kolasa-Raczka A, Kalinowski P. Dental age in patients with impacted maxillary canines related to the position of the impacted teeth. Eur. J. Orthod. 33, 2011, No 5, 492-497.

\section{Corresponding author:}

Dr. Hristina Arnautska, D.M.Sc.

Department of PDM and Orthodontics, Faculty of Dental Medicine, MU - Varna

84 Tsar Osvoboditel Street

Varna, Bulgaria

mobile phone: +359877599675

e-mail: tineia@yahoo.com 